Präv Gesundheitsf 2021 • 16:123-130 https://doi.org/10.1007/s11553-020-00799-5 Eingegangen: 16. April 2020

Angenommen: 6 . Juni 2020

Online publiziert: 26. Juni 2020

(c) Der/die Autor(en) 2020

\author{
Annika Wilke ${ }^{1,2}(\mathbb{D}) \cdot$ Andreas Hansen $^{1,2}(\mathbb{D}) \cdot$ Michaela Ludewig $^{1,2}(\mathbb{D}) \cdot$ \\ Marc Rocholl ${ }^{1,2}$ (D) - Swen Malte John ${ }^{1,2}$ iD \\ 'Abteilung Dermatologie, Umweltmedizin und Gesundheitstheorie, Institut für Gesundheitsforschung \\ und Bildung, Universität Osnabrück, Osnabrück, Deutschland \\ ${ }^{2}$ Institut für interdisziplinäre Dermatologische Prävention und Rehabilitation (iDerm) an der Universität \\ Osnabrück, Osnabrück, Deutschland
}

\title{
Prävention berufsbedingter Erkrankungen durch Lehrkräfte
}

\section{Entwicklung von Unterrichtsmaterialien am Beispiel von Hauterkrankungen in Epoxidharz-verarbeitenden Ausbildungsberufen}

\begin{abstract}
Lehrkräfte an Berufsschulen können als Multiplikator*innen fungieren, damit Schüler*innen bereits während der Ausbildung lernen, die eigene Haut am Arbeitsplatz zu schützen sowie Erkrankungen und einer möglichen Berufsaufgabe vorzubeugen. Für den Unterricht bereitgestellte Materialien sollten den Bedürfnissen und Anforderungen der Zielgruppe "Lehrkräfte“ entsprechen, um Anwendung zu finden.
\end{abstract}

\section{Hintergrund und Fragestellung}

Berufsbedingte Hauterkrankungen sind die häufigsten Erkrankungen, die jährlich den Unfallversicherungsträgern in Deutschland gemeldet werden [4]. Diese können zu Einschränkungen der Lebensqualität, hohen Kosten und zur Berufsaufgabe führen $[1,13]$. Der Hautkontakt zu verschiedensten beruflichen Irritanzien und Allergenen kann diese Erkrankungen auslösen [9]. So sind beispielsweise Epoxidharze hochpotente Allergene, die bei unsachgemäßem Umgang nach kurzer Zeit zu einer Sensibilisierung und einem allergischen Kontaktekzem der Haut führen können, was Betroffene dazu zwingen kann, den Beruf aufzugeben $[5,8,25]$. Epoxidharze werden aufgrund ihrer besonderen, technischen Ei- genschaften in vielen Berufsfeldern eingesetzt $[5,8,26]$ und können häufig nicht durch andere Stoffe ersetzt werden $[5,8]$. Daher kommt technischen und organisatorischen Schutzmaßmaßen und die Verwendung persönlicher Schutzausrüstung (PSA) am Arbeitsplatz große Bedeutung $\mathrm{zu}$.

Idealerweise sollten hautschonende Arbeitsweisen und die korrekte Anwendung von PSA bereits beim Erlernen einer beruflichen Tätigkeit als selbstverständlicher Teil der Berufsausübung internalisiert werden. Im Rahmen der dualen Ausbildung in Deutschland ist daher die Berufsschule - neben dem Lernort „Betrieb“ - ein bedeutsames Setting. Lehrkräfte an Berufsschulen können somit in der Prävention als Multiplikatorinnen für Auszubildende in exponierten Risikoberufen fungieren. Multiplikator*innen sollen im Folgenden gemäß Blümel und Lehmann [2] verstanden werden als „Personen oder Institutionen, die empfangene Informationen an mehrere Personen (i.d.R. die Zielgruppe) weiterleiten und dadurch multiplizieren“.

Vor diesem Hintergrund wurde das Projekt FF-FP 0401 „Prävention von Berufsdermatosen bei Auszubildenden mit Kontakt zu Epoxidharzen (EpoxSafe@School 1.0)“ durchgeführt, das von der Deutschen Gesetzlichen Unfall- versicherung (DGUV) gefördert wurde [27]. Das Projektziel war die Konzeption von Unterrichtsmaterialien für Auszubildende mit Kontakt zu Epoxidharzen, die auf dem aktuellen wissenschaftlichen Erkenntnisstand basieren und von Lehrkräften an Berufsschulen eingesetzt werden können. Der Einsatz von Unterrichtsmaterialien zu diesem Themenfeld soll von den Lehrkräften jedoch nicht als zusätzliche Belastung bzw. Aufgabe wahrgenommen werden. Dem Projekt lag daher - in Anlehnung an die Leitgedanken der guten gesunden Schule [18, 19] - der Ansatz zugrunde, die Materialien explizit auf die Bedürfnisse der Zielgruppe "Lehrkräfte" abzustimmen, damit diese von Lehrkräften als Beitrag zur Umsetzung des Bildungsauftrags der Berufsschule und als gewinnbringend für den Arbeitsalltag wahrgenommen werden. Im Rahmen des Projekts stellte sich daher die zentrale Frage, welche Anforderungen von Lehrkräften an Berufsschulen an fremd entwickelte Unterrichtsmaterialen gestellt werden, und wie die Materialien aufzubauen sind. Die zentralen Projektergebnisse zur Beantwortung dieser Fragestellung und zur Entwicklung der Materialien werden nachfolgend dargestellt. 
Tab. 1 Auszug aus dem Moderationsleitfaden für die Fokusgruppendiskussionen

Hauptfragenkatalog - Anforderungen und Bedürfnisse an Materialien

Bitte denken Sie einmal an Unterrichtsmaterialien, die nicht von Ihnen selbst, sondern von fremden Personen oder Institutionen entwickelt worden sind: Was sind Kriterien oder Merkmale, die dazu führen, dass Sie Materialien definitiv nicht in Ihren eigenen Unterricht integrieren werden?

Und nun möchten wir Sie bitten, sich diese Kriterien anzusehen und positiv zu formulieren, was "gute" Unterrichtsmaterialien ausmachen muss, damit Sie sie in ihrer eigenen Unterrichtspraxis einsetzen.

Ggf. nachfragen: bestimmte Methoden/Medien/Aktions-/Sozialformen

Bitte denken Sie nochmal explizit an das Thema „Prävention von Hauterkrankungen im Umgang mit Epoxidharzen": Gibt es hier besondere Merkmale, die "gute" Unterrichtsmaterialien kennzeichnen?

\section{Methodik}

\section{Allgemeines Vorgehen}

Die Schritte des konzeptionellen Projektes orientierten sich an den Abläufen, die von Craig et al. [3] und Lenz [14] für komplexe Interventionen sowie von Meisert [16] für (Biologie-)Unterricht formuliert wurden. Das Projekt besteht aus drei Phasen:

- Phase 1: „Identifikation der Evidenzbasis“" (z.B. Recherche und Aufarbeitung der Literatur zum Themengebiet „Epoxidharze“, Analyse des Settings „Berufsschule“ und der curricularen Rahmenbedingungen),

- Phase 2: „Identifizieren einer Wirktheorie" (z. B. Wahl des Health Action Process Approaches (HAPA-Modell; [24]) als theoretisches Rahmenmodell zur Erklärung gesundheitsrelevanten Verhaltens) und

- Phase 3: „Modellierung der Prozesse und der Outcomes“ (z. B. Fokusgruppendiskussionen mit Lehrkräften, didaktische Konstruktion der Materialien).

\section{Literaturrecherche}

Im Februar 2017 erfolgte eine Literaturrecherche in den Datenbanken Medline (via PubMed), CISDOC, NIOSHTIC-2, Forschungsdatenbank Arbeitsschutz sowie in einschlägigen Fachzeitschriften zum Themenfeld "Epoxidharze“. Deutsch- und englischsprachige Publikationen sowie alle Publikationsformate und Studiendesigns wurden eingeschlossen. Die Ergebnisse bildeten die Basis für: a) die Aufarbeitung der Epidemiologie zur Identifikation von Risikoberufen für die Wahl der Bildungsgänge,

b) die Identifikation von Risikotätigkeiten und Arbeitsabläufen für die Formulierung von Lernsituationen (s. unten),

c) die Inhalte der Unterrichtsmaterialien hinsichtlich der Entstehung von berufsbedingten Hauterkrankungen sowie der sicheren Verarbeitung von Epoxidharzen.

Ferner wurden ergänzende Online-Recherchen (z. B. bei Unfallversicherungsträgern) durchgeführt, um Broschüren und andere Praxismaterialien zu identifizieren.

\section{Fokusgruppendiskussionen}

Damit Materialien von Lehrkräften eingesetzt werden, sind diese an die Bedürfnisse, Erwartungen und die alltägliche Lebenswelt dieser Zielgruppe anzupassen. Um die Perspektive potenzieller Multiplikator*innen bei der didaktischen Konstruktion zu berücksichtigen, wurden Fokusgruppendiskussionen mit Lehrkräften berufsbildender Schulen geplant. Diese moderierten Diskussionen in Kleingruppen dienen nicht der Konsensfindung und der Ermittlung repräsentativer Aussagen, sondern zielen darauf ab, möglichst viele Facetten und Meinungen und erfahrungsbasierte Einstellungen zu einem Thema bzw. zu einer oder mehrerer konkreter Fragestellungen in einer Zielgruppe zu explorieren [12, 22, 23].

Die Durchführung der Fokusgruppendiskussionen wurden im Mai 2017 von der Landesschulbehörde Niedersachsen genehmigt. Im Anschluss wur- den niedersächsische Berufsschulen im Raum Osnabrück mit geeigneten Bildungsgängen, d.h. Berufsfeldern, in denen Epoxidharze verarbeitet werden, kontaktiert. Sofern das Einverständnis von Seiten der Schulleitungen vorlag, erfolgte die Kontaktaufnahme mit den Abteilungsleitern relevanter Ausbildungsgänge, mit denen - bei Interesse - die Fokusgruppen konkret geplant wurden (z.B. Terminfindung, Akquise von Lehrkräften).

Für die Durchführung wurde ein teilstrukturierter Moderationsleitfaden entwickelt (• Tab. 1), um insbesondere zu ermitteln, welche Anforderungen Lehrkräfte an fremd entwickelte Unterrichtsmaterialen stellen und wie diese gestaltet sein sollten, um eingesetzt $\mathrm{zu}$ werden. Ferner wurden Beispiele für Unterrichtsmaterialien, z.B. ein Experiment, um die Permeation von Chemikalien durch einen Schutzhandschuh $\mathrm{zu}$ demonstrieren, vorgestellt und Eindrücke, Fragen sowie Vor- und Nachteile diskutiert (• Abb. 1).

Ein Moderator (AH) leitete die Diskussion, eine Moderatorin (AW) notierte die genannten Aspekte stichwortartig auf Moderationskarten und Flipcharts. Am Ende eines Themenblockes fasste die Moderatorin die genannten Aspekte im Sinne der kommunikativen Validierung zusammen. Ergänzend fertigte eine studentische Mitarbeiterin während der gesamten Fokusgruppendiskussionen ein Protokoll an und die Diskussionen wurden mithilfe von zwei digitalen Diktiergeräten (Olympus VN-8600PC, Olympus, Hamburg, Deutschland) aufgenommen. Abschließend füllten die teilnehmenden Lehrkräfte einen Kurzfragebogen zu demographischen Angaben und zur Berufserfahrung aus. Die digitalen Audioaufnahmen wurden mit dem Programm easytranscript (e-werkzeug, Berlin, Deutschland) mit einem vereinfachten Transkriptionssystem [7] verschriftlicht und in Anlehnung an die strukturierende qualitative Inhaltsanalyse nach Mayring [15] mit der Software QCAmap (Verein zur Förderung Qualitativer Forschung ASQ, Klagenfurt, Österreich) ausgewertet. Die teilnehmenden Lehrkräfte wurden über den Datenschutz mündlich und schriftlich aufgeklärt und 
Präv Gesundheitsf 2021 · 16:123-130 https://doi.org/10.1007/s11553-020-00799-5

(c) Der/die Autor(en) 2020

A. Wilke · A. Hansen · M. Ludewig · M. Rocholl · S. M. John

\section{Prävention berufsbedingter Erkrankungen durch Lehrkräfte. Entwicklung von Unterrichtsmaterialien am Beispiel von Hauterkrankungen in Epoxidharz-verarbeitenden Ausbildungsberufen}

\section{Zusammenfassung}

Hintergrund. Aufgrund ihrer technischen Eigenschaften werden Epoxidharze (Kunstharze) in vielen Berufen eingesetzt. Wegen ihres hohen Allergiepotenzials ist ein adäquates Hautschutzverhalten bei der Verarbeitung von hoher Bedeutung. Lehrkräfte an Berufsschulen können als Multiplikator*innen fungieren, damit Auszubildende bereits beim Erlernen eines Berufs die notwendigen Kenntnisse und Fertigkeiten erwerben und Hautschutz als selbstverständlichen Teil der Arbeitsroutinen internalisieren.

Ziel der Arbeit. Das konzeptionelle Projekt „EpoxSafe@School“ zielte darauf ab, Unterrichtsmaterialien zur Prävention epoxidharzbedingter Hauterkrankungen für die Berufsschule zu entwickeln, die auf dem wissenschaftlichen Erkenntnisstand basieren und an die Bedürfnisse von Lehrkräften angepasst sind.

Material und Methoden. Im Projektverlauf wurden die Anforderungen und Bedürfnisse von Lehrkräften an durch fremde Personen erstellte Unterrichtsmaterialien im Rahmen von zwei Fokusgruppendiskussionen erhoben. Die Ergebnisse wurden bei der didaktischen Konstruktion der Materialien berücksichtigt. Ferner erfolgten Recherchen (z. B. zur Evidenz, zum Setting Berufsschule) als Grundlage für die Entwicklung der Materialien. Ergebnisse und Diskussion. Von den Lehrkräften wurden folgende Anforderungen an fremd erstellte Materialien formuliert: methodische Variabilität, Flexibilität und Veränderbarkeit, Exemplarität, Alltagsnähe, Berufsbezug sowie die Berücksichtigung des curricularen Rahmens. Die vor diesem Hintergrund entwickelten Materialien (z. B. Schüler-/Lehrertexte, Arbeitsblätter, Experimente) sind seit Projektende frei verfügbar. Ausblickend sind Untersuchungen zur Bewertung der Materialien durch Lehrkräfte und Auszubildende sowie zum Praxiseinsatz und deren möglicher Effekte empfehlenswert.

Schlüsselwörter

Kontaktekzem · Schule · Fokusgruppen .

Deutschland $\cdot$ Schutzhandschuhe

\section{Prevention of work-related diseases by teachers. Development of teaching materials concerning skin diseases using the example of epoxy resin processing training professions in vocational schools}

\section{Abstract}

Background. Due to their technical properties, epoxy resins (synthetic resins) are used in many occupations. Because of their high allergy potential, adequate skin protection during processing is of great importance. Teachers at vocational schools can act as multipliers so that trainees gain the necessary knowledge and skills while learning a profession and internalize skin protection as a natural part of work routines.

Objectives. The conceptual project "EpoxSafe@School" aimed to develop teaching materials for the prevention of epoxy resinrelated skin diseases for vocational schools, based on the state of scientific knowledge and adapted to the needs of teachers.

Materials and methods. In the course of the project, the requirements and needs of teachers with regard to teaching materials produced by third parties were surveyed in two focus group discussions. The results were taken into account in the didactic construction of the materials. Furthermore, research (e.g., on evidence, on the setting of vocational schools) was carried out as a basis for the development of the materials.

Results and conclusions. The teachers specified the following requirements for materials developed by third parties: methodological variability, flexibility and changeability, exemplariness, closeness to everyday life, occupational relevance, and consideration of the curricular framework. The materials developed against this background (e.g., pupil/teacher texts, worksheets, experiments) have been freely available since the end of the project. For the future, studies on the evaluation of the materials by teachers and trainees as well as on the practical application and their possible effects are recommended.

Keywords

Contact Dermatitis - School - Focus groups . Germany $\cdot$ Protective gloves haben eine schriftliche Einverständniserklärung unterschrieben.

\section{Ergebnisse}

\section{Rahmenbedingungen des Unterrichts}

Für eine hohe Passgenauigkeit der Materialien für das Setting „Berufsschule“ wurden die curricularen Rahmenbedingungen analysiert, die von den Lehrkräften bei der Unterrichtsplanung zu beachten sind. So wurden Rahmenlehrplä- ne (RLP) als Ordnungsmittel berücksichtigt sowie das seitens der Kultusministerkonferenz (KMK) geforderte Bildungsziel der Berufsschule („Erwerb beruflicher Handlungskompetenz“; [11, 21]).

Berufsschulischer Unterricht erfolgt in Lernfeldern (LF), d.h. „(...) thematische(n) Einheiten, die an beruflichen Aufgabenstellungen und Handlungsfeldern orientiert sind und den Arbeitsund Geschäftsprozess reflektieren“ [10]. Im Unterricht soll berufliche Handlungskompetenz ausgehend von konkreten beruflichen Situationen durch die Bearbei- tung von Lernsituationen handlungsorientiert erworben werden $[10,21]$. Lernsituationen wiederum sollen bestimmte Merkmale erfüllen, z. B. Berufsorientierung (Gegenwarts- bzw. Zukunftsbedeutung) sowie Exemplarität und Übertragbarkeit auf andere berufliche Situationen [17]. Für das Projekt wurde die Implikation abgeleitet, Lernsituationen als Unterrichtseinstiege zu erstellen, die inhaltlich und methodisch diese Aspekte berücksichtigen. 
Ziel: „Die unsichtbare Gefahren sichtbar machen", d. h. das Durchwandern von Chemikalien (z. B. Epoxidharze, Lösemittel) auf molekularer Ebene durch flüssigkeitsdichte Handschuhe (Permeation)

\section{Konsequenz für Hautschutz am Arbeitsplatz:}

Flüssigkeitsdichte „Chemikalienschutzhandschuhe“ müssen konsequent und rechtzeitig gewechselt werden.
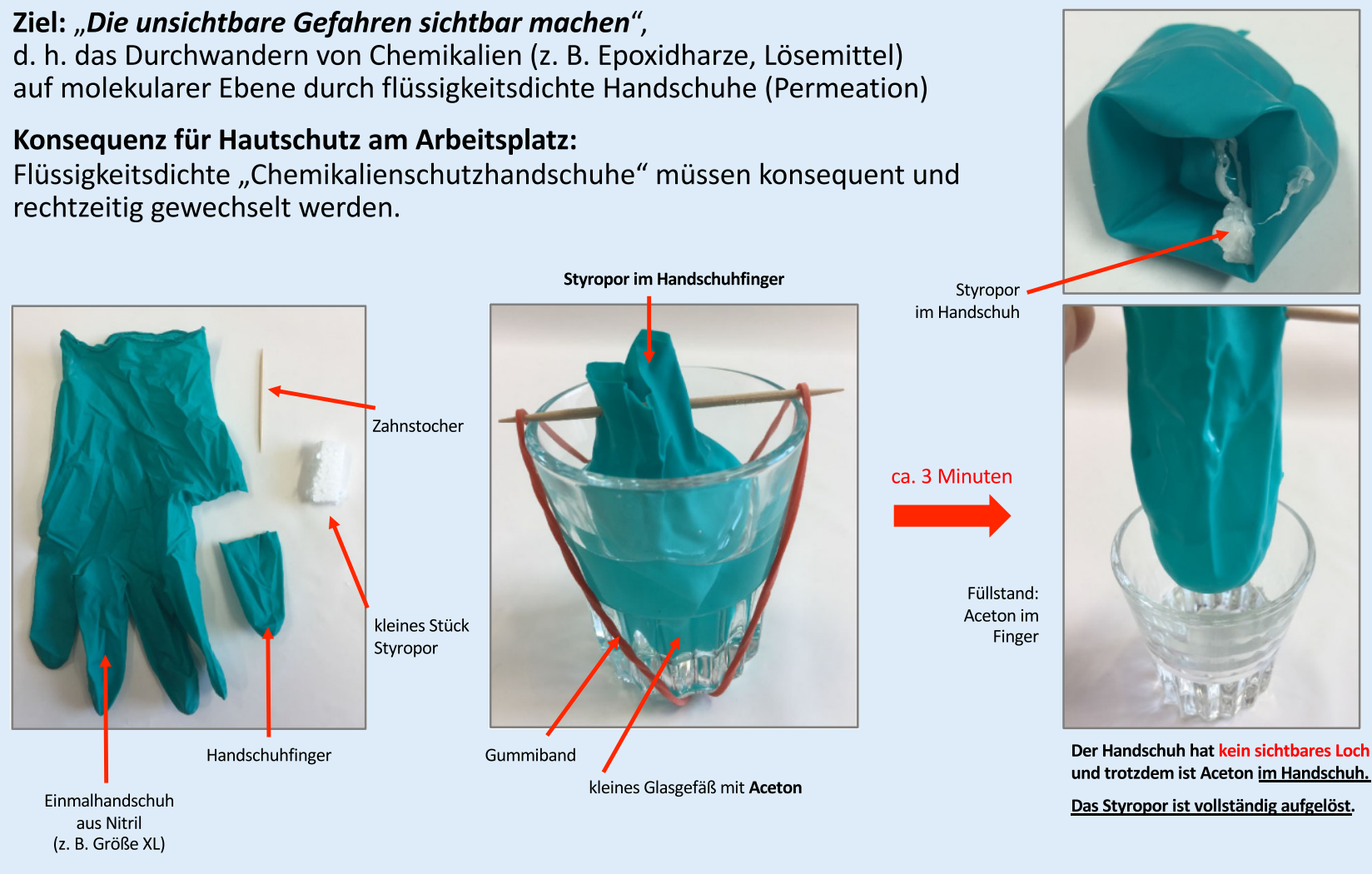

Abb. 1 ॥ Material zur Diskussion im Rahmen der Fokusgruppen: Experiment zur Visualisierung von - ansonsten unsichtbarer - Permeation von Chemikalien (hier: Aceton) durch Handschuhe (hier: Nitril-Einmalhandschuh)

\section{Wahl der Ausbildungsberufe}

Die Materialien sollen breit in verschiedenen RLP und Ausbildungsberufen einsetzbar sein, wobei der Lehrkraft die Entscheidung obliegt, wann die Inhalte in welchen Bildungsgang integriert werden. Zum Recherchezeitpunkt standen seitens der KMK 322 RLP für anerkannte Ausbildungsberufe zur Verfügung, von denen zunächst Berufe ausgeschlossen wurden, in denen ein Epoxidharzkontakt nicht zu erwarten ist, z. B. kaufmännische Berufe. Auf der Basis der Literaturrecherche wurden aus den übrigen 143 Berufen 40 Ausbildungsberufe für die Entwicklung von Lernsituationen kriteriengeleitet ausgewählt, z. B. solche, bei denen es sich um Risikoberufe mit hoher Prävalenz von Epoxidharzsensibilisierungen handelt (z. B. „Fliesen-, Platten- und Mosaikleger*in“).

\section{Curriculare Verortung}

Die Materialien können curricular grundsätzlich im Kontext „Arbeits-/ Gesundheitsschutz/Arbeitsplatzhygiene" jedes Ausbildungsgangs verortet werden, wobei die Verarbeitung von Epoxidharzen als exemplarische Tätigkeit dient, um übergreifende Inhalte und Prinzipien des Arbeitsschutzes zu erlernen (z.B. Auswahl und Verwendung von PSA). Ferner wurden Schlagworte für typische Tätigkeiten mit Epoxidharzkontakt formuliert, die im RLP auf einen entsprechenden Arbeitsprozess hinweisen (z. B. „Anstrich“, „Epoxid“, „Estrich“, „laminieren“), um adäquate Verortungsmöglichkeiten im RLP zu identifizieren.

\section{Fokusgruppendiskussionen}

\section{Teilnehmende}

Es wurden Fokusgruppendiskussionen $(F)$ an zwei berufsbildenden Schulen durchgeführt (F1: 04.09.2017, Dauer: $2 \mathrm{~h}$ 3 min; F2: 05.09.2019, Dauer: 2 h 4 min).
F1 setzte sich aus vier männlichen Lehrkräften zusammen, von denen drei im Bereich Bautechnik unterrichteten und einer im Bereich Holztechnik. Im Durchschnitt arbeiteten die Lehrer seit knapp 10 Jahren in diesem Beruf (Minimum: 4 Jahre, Maximum 14 Jahre). F2 bestand aus drei männlichen Lehrkräften. Zwei Teilnehmer unterrichteten im Bereich Bautechnik, einer im Bereich Holztechnik. Im Durchschnitt arbeiteten diese Teilnehmer seit 23 Jahren als Lehrkraft (Minimum: 12 Jahre, Maximum 30 Jahre).

\section{Anforderungen an fremd erstellte Unterrichtsmaterialien}

Aus F1 und F2 wurden Anforderungskriterien abgeleitet, anhand derer Lehrkräfte fremd zur Verfügung gestellte Unterrichtsmaterialien bewerten bzw. die die Verwendung von Materialien im Unterricht beeinflussen. Zentrale Aspekte werden nachfolgend dargestellt. 


\section{Zeitersparnis}

Fremde Unterrichtsmaterialien sollten eine zeitliche Ersparnis gegenüber selbst entwickelten Materialien bieten. Dies gilt u.a. bei der Vorbereitung eines fachfremden Themas. Hier sollten zentrale Hintergrundinformationen knapp zusammengefasst und Musterlösungen bereitgestellt werden. Gut aufbereitete Materialien und Arbeitsblätter erscheinen wichtiger als umfangreiche didaktische Kommentare.

„Also es muss irgendwie schon ein Zeitgewinn bei mir rumkommen, oder halt irgendwie dass ich, ja so erstmal nicht in der Lage gewesen wäre, weil ich da nicht so in der Thematik bin, was halt dieses medizinische Wissen angeht, überhaupt die Schüler da ordentlich ranzuführen" (F1: Lehrer A; Z: 618-621).

\section{Methodische Variabilität}

Die Materialien sollten methodische Variabilität und Variationsmöglichkeiten (z.B. hinsichtlich der Sozialform) bieten, damit der Unterricht an die jeweilige Zielgruppe - z.B. hinsichtlich Ausbildungsgang und Bildungsvoraussetzungen - angepasst werden kann. So können beispielsweise Rollenspiele eine geeignete Zugangsmöglichkeit $\mathrm{zu}$ bestimmten Themenbereichen sein, z. B. in Form eines Argumentationstrainings zur Verwendung von Schutzhandschuhen bei der Arbeit. Rollenspiele sollten jedoch nicht den gesamten Unterricht dominieren.

„Zu viel Text, schreckt gerade die Zielgruppen $a b$, in denen ich da bin." (F1: Lehrer B; Z: 69-71).

\section{Flexibilität und Veränderbarkeit}

Die Materialien sollten flexibel und modular an die Bedürfnisse und den Stil der einzelnen Lehrkraft anpassbar sein, ähnlich eines Baukastenprinzips. Flexibel einsetzbare Materialien sollen es ermöglichen, dass der Unterricht und damit verbundene Entscheidungen $\mathrm{zu}$ Inhalten und Methoden zum „eigenen“ Unterricht gemacht werden kann. Dies bedeutet auch, dass Materialien anpassbar und veränderbar sein sollten. So erscheint es wünschenswert, sie in einem Dateiformat zur Verfügung zu stellen, das veränderbar ist (z. B. Word statt pdf).

„Schön finde ich bei sowas auch, wenn ich es auf mich anpassen kann. Wenn ich es jetzt nicht in einem Format kriege, das ich nicht mehr ändern kann" (F1: Lehrer B; Z: 300-301).

\section{Exemplarität}

Wenn Inhalte exemplarisch nutzbar und auf andere Themenfelder übertragbar sind, kann dies einen höheren (zeitlichen) Aufwand rechtfertigen, z.B. ein Experiment zur Permeation von Chemikalien durch Handschuhe, das auf andere Bereiche der persönlichen Schutzausrüstung übertragbar ist.

„Und wenn das eben dieses Exemplarische ist, für ich sag mal $x$ andere Fälle, ob ich den richtigen Atemschutzfilter habe bei Atemschutz, ob ich den richtigen Gehörschutzfilter habe, bestimmte Frequenzen, oder was weiß ich, oder Staubschutz für bestimmte Stäube und so was, dann ist es so neunzig Minuten sind da ja auch total effektiv untergebracht" (F1: Lehrer C; 960-964).

\section{Realitäts-/Alltagsnähe und Berufsbezug}

Materialien sollen zielgruppenorientiert, realistisch und praxisnah, d.h. an die berufliche Lebenswelt der Auszubildenden angepasst sein. Auf realitätsferne Darstellungen (z.B. allzu saubere Arbeitskleidung bei verschmutzenden Tätigkeiten) sollte verzichtet werden. Alltagsnahe Fallbeispiele können für einen angemessenen Berufsbezug ein geeignetes Mittel darstellen, wobei fehlerhafte Arbeitsabläufe Potenzial zum Lernen bieten können.

„Ich könnte mir auch vorstellen, aus Fehlern zu lernen, also dass man auch vielleicht ein Fallbeispiel konstruiert, was natürlich auch aus der Praxis kommt" (F2: Lehrer 2; Z: 450-451).

\section{Experimente}

In F1 und F2 wurde ein Experiment als konkretes Beispiel für ein Unterrichtselement diskutiert (• Abb. 1). Hierzu ließen sich unterschiedliche Einschätzungen er- kennen: Experimente könnten Interesse und Neugier wecken und Inhalte blieben $u$.U. durch die Visualisierung bei den Schüler*innen länger in Erinnerung. Beim Einsatz von Experimenten scheint ein entscheidendes Kriterium zu sein, dass diese einfach und gefahrlos durchführbar sind. So erschien die Durchführung des vorgestellten Experimentes mit Aceton aus Sicht der Lehrkräfte unter anderem davon abhängig, ob geeignete Räumlichkeiten (z.B. ein Chemieraum) zur Verfügung stehen.

"Also so wie das dargestellt ist, ist es schon sehr anschaulich. Also eine praktische Umsetzung des Experimentes bei uns sehe ich so nicht. Es gibt zum Beispiel bei uns an der berufsbildenden Schule keinen Chemieunterricht mehr" (F1: Lehrer D; Z: 826-828).

Ein Lehrer der anderen Schule beurteilt die Durchführung hingegen aufgrund der verfügbaren Ressourcen als leicht umsetzbar, da hier ein geeigneter Laborraum zur Verfügung stünde:

„Kann ich morgen früh mit anfangen. Alles habe ich da, also" (F2: Lehrer 1; 1410).

Im Umgang mit Gefahrstoffen sei jedoch Vorsicht geboten und Rücksprache mit einem Fachlehrer der Chemie sei u. U. erforderlich.

Als mögliche Alternative zur Durchführung vor Ort wurde ein Video des Experiments genannt. Ferner könnten Experimente je nach Zielgruppe als Lehrerexperiment statt als Schülerexperiment durchgeführt werden.

\section{Rahmenbedingungen der Berufsschule}

In F1 und F2 gaben Lehrkräfte übergeordnete Aspekte zu den curricularen Rahmenbedingungen an, die für die Unterrichtsinhalte und damit auch die Materialien von Bedeutung sind. So sollte das Material beispielsweise den curricularen Vorgaben entsprechen.

„Also schön wär es, wenn es in die Schemata von den Vorgaben des Landes passt" (F1; Lehrer D; Z: 1570-1571).

Ferner gab eine Lehrkraft an, eine Verdichtung des Unterrichts in Form eines steigenden Umfangs der zu behandeln- 
den Inhalte in der Ausbildung zu bemerken.

"Als ich angefangen habe, Tischler zu lernen, gab es vom Europa Lehrmittelverlag ein drei Zentimeter dickes Buch. Mittlerweile ist das Buch sechs Zentimeter dick. Die Schüler lernen heute nicht schneller als wir, sind einfach nur viel mehr Inhalte, die die heute vermittelt kriegen. Keiner streicht irgendwas raus, was weg bleibt. Alle schreiben immer was Neues dazu" (F2, Lehrer 1; Z: 1636-1640).

\section{Didaktische Konstruktion der Materialien}

Die Ergebnisse von F1 und F2 wurden bei der didaktischen Konstruktion wie folgt berücksichtigt:

\section{Lernziele und Themenblöcke}

Zunächst wurden Lernziele (z.B. „Die Schülerinnen und Schüler unterscheiden Handschuhmaterialien und -modelle und wählen geeignete Handschuhe exemplarisch für die Verarbeitung epoxidharzhaltiger Berufsstoffe aus.") als Grundlage für die didaktische Konstruktion sowie vier Themenblöcke (z. B. Block 4: Hautschutz planen und kommunizieren) definiert.

\section{Methodische Variabilität}

Es wurden Materialien entwickelt, die eine unterschiedliche methodische Erarbeitung der Themen ermöglichen, z.B. Lernsituationen, Schülertexte, ein Fallbeispiel (als Text- und Audiodatei), Arbeitsblätter (z.B. zur Planung des eigenen Hautschutzes, zur Durchführung Rollenspielen zur Thematisierung von Hautschutz aus unterschiedlichen Perspektiven), Foliensätze sowie Experimente (z.B. als Lehrer- oder Schülerexperiment, teils als Variante mit weniger gefährlichen Chemikalien, teils als Videos). Auf ausführliche didaktische Kommentare für Lehrkräfte wurde zugunsten knapper Hintergrundinformationen verzichtet.

\section{Flexibilität und Veränderbarkeit}

Es wurde keine feststehende Lerneinheit entwickelt, die in einem Standardformat mit festgelegter Dauer und Reihenfolge durchzuführen ist, sondern Materialien, die flexibel und modular einsetzbar sind und an die jeweilige Zielgruppe und die Bedürfnisse bzw. den Unterrichtsstil der Lehrkraft angepasst werden können. Dies greift den Wunsch auf, dass Unterricht zum „eigenen Unterricht“ der Lehrkraft werden soll. Dem Wunsch nach Veränderbarkeit und Anpassungsmöglichkeit der Materialien wurde durch die Bereitstellung von „offenen“ Wordund PowerPoint-Dateien entsprochen, die Lehrkräfte bedarfsorientiert modifizieren können.

\section{Exemplarität}

Verschiedene Inhalte der Materialien weisen Exemplarität für andere Themenfelder auf. So kann beispielsweise das Phänomen der „Permeation“ im Kontext von Arbeits- und Hautschutz exemplarisch auf andere Bereiche der persönlichen Schutzausrüstung übertragen werden. Daher wurde speziell für dieses Thema ein Experiment mit verschiedenen Variationsmöglichkeiten zur Visualisierung von Permeation entwickelt.

\section{Realitäts-/Alltagsnähe und Berufsbezug}

Ein hoher Berufs- und Alltagsbezug wurde u. a. umgesetzt, indem die Lernsituationen als Möglichkeit des Unterrichtseinstiegs durch berufstypische Tätigkeiten mit Epoxidharzkontakt an die Lebenswelt der Auszubildenden angepasst wurden. Die Aktualität von Produktionsverfahren und Produkten wurde von Mitgliedern des Arbeitskreises Epoxidharze (z. B. Mitarbeiter ${ }^{\star}$ innen verschiedener Unfallversicherungsträger) geprüft.

Ein persönlicher Bezug wird durch die Integration eines realen Fallbeispiels (junger Auszubildender, der seine Ausbildung aufgrund einer Epoxidharzallergie abbrechen musste) in die Materialien integriert, mit dem sich die Schüler*innen hinsichtlich Alter und Beruf sowie potenzieller persönlicher Konsequenzen identifizieren können.

\section{Curricularer Rahmen der Berufsschule}

Die Materialien wurden auf den curricularen Rahmen der Berufsschule abge- stimmt, indem u. a. konkrete Vorschläge zur Verortung in verschiedenen Ausbildungsjahren und Lernfeldern - und damit im jeweiligen RLP - gemacht wurden. Bei den Lernsituationen handelt sich um komplexe, berufstypische Situationen (Berufsorientierung). Sie ermöglichen eine vollständige Handlung und erlaubt den Erwerb von beruflicher Handlungskompetenz, um ein Problem zu lösen, das berufliche, persönliche, soziale und gesellschaftliche Dimensionen aufweist, was dem Bildungsziel des handlungsorientierten Unterrichts entspricht.

\section{Dissemination}

Die Materialien wurden im Schulportal der Deutschen Gesetzlichen Unfallversicherung (DGUV) „Lernen und Gesundheit" (LuG) eingestellt (URL: www. dguv-lug.de, Webcode: lug1001301). Verlinkungen wurden beim Portal für Unterrichtsmaterialien „Lehrer Online“ (URL: www.lehrer-online.de) und bei der Bundesanstalt für Arbeitsschutz und Arbeitsmedizin (Baua; URL: www.baua. de/epoxidharze) erstellt.

Von März 2019 bis November 2019 (9 Monate) konnten für die LuG-Website mit den EpoxSafe-Materialien insgesamt 2097 Zugriffe verzeichnet werden ${ }^{1}$. Für die dort unter sieben verschiedenen Links angebotenen Lehrmaterialien wurden insgesamt 2591 Downloads verzeichnet, sodass davon auszugehen ist, dass nicht jeder Besucher alle Lehrmaterialien herunterladen, sondern eine gezielte Auswahl getroffen wird. Die Zahlen liegen nach Aussage des Plattformbetreibers im normalen Durchschnitt aller Unterrichtseinheiten ${ }^{2}$.

\section{Diskussion}

Im Rahmen des konzeptionellen Projekts „EpoxSafe@School“ wurden Unterrichtsmaterialien für Lehrkräfte an Berufsschulen zur Prävention von berufsbedingten Hauterkrankungen in epoxid-

\footnotetext{
1 Lernen und Gesundheit (2020) Das Schulportal der Deutschen Gesetzlichen Unfallversicherung (DGUV). Universum Verlag, Wiesbaden (persönliche Kommunikation im Februar 2020) 2 ebd.
} 
harzverarbeitenden Ausbildungsberufen entwickelt. Im Rahmen von zwei Fokusgruppendiskussionen wurden Anforderungen und Bedürfnisse von Lehrkräften an von fremden Personen erstellte Unterrichtsmaterialien ermittelt und bei der Konzeption der Materialien berücksichtigt, z. B. methodische Variabilität, Veränderbarkeit und Alltagsnähe.

Lehrkräfte sind Multiplikator*innen, die aufgrund ihrer Bildungsbiographie für die Planung und Durchführung von Unterricht für verschiedene Zielgruppen ausgebildet sind. Sie können daher modular anwendbare Materialien den Rahmenbedingungen des Unterrichts entsprechend auswählen und ggf. adaptieren. Es ist anzunehmen, dass sich Lehrkräfte in diesem Punkt von anderen, in der (Primär)prävention tätigen Multiplikatorengruppen ohne entsprechende pädagogische Ausbildung und Berufserfahrung unterscheiden. Für diese Gruppen könnten standardisierte(re) Unterrichts- bzw. Schulungs- oder Beratungseinheiten Vorteile aufweisen.

Als Stärke des Projekts ist zu nennen, dass im Projektverlauf eine unmittelbare Verzahnung zwischen der Bedarfsermittlung in der Zielgruppe "Lehrkräfte“ und der didaktischen Konstruktion der Materialien erfolgte.

Die Anzahl der Fokusgruppendiskussion und der Teilnehmenden ist als eine Limitation zu nennen. Gleichwohl findet sich Literatur zur qualitativen Forschung mit homogenen Teilnehmergruppen, die zwei Fokusgruppen [20] mit 4-8 Teilnehmenden [6] als Mindestanforderungen formuliert. Die geringe Anzahl der Fokusgruppen und Teilnehmenden im vorliegenden Projekt ist auf die Rekrutierung von Schulen und Lehrkräften zurückzuführen, die sich als herausfordernd dargestellt hat. Eine Unterschätzung der Bedeutung des Themas und fehlende Zeit für eine Auseinandersetzung mit dem Projekt können mögliche Gründe sein. Künftig sollten hier andere, ggf. noch persönlichere Rekrutierungsstrategien erprobt werden.

Grenzen und gleichzeitig Ansatzpunkte für weiterführende Forschungsprojekte ergeben sich, da die abschließende Bewertung der erstellten Materialien durch Lehrkräfte (z. B. hinsichtlich der tatsächlichen Verwendbarkeit in der Unterrichtspraxis) nicht Teil des Projekts war. Da das Projekt ausschließlich die Entwicklungsphase einer komplexen Intervention umfasste, könnte dies im Rahmen nächster Schritte erfolgen (z. B. im Rahmen einer Machbarkeitsprüfung und Pilotierung; $[3,14])$.

Anhand der Zugriffs- und Downloadzahlen können keine Aussagen über die tatsächliche Nutzung in der Praxis getroffen werden. Jedoch ist es als positiv zu werten, dass die Zahlen - trotz des vergleichsweise speziellen Themenfelds - im durchschnittlichen Bereich liegen. Zudem ist abzuleiten, dass die Materialien - wie intendiert - gezielt ausgewählt und genutzt werden und die wenigsten Besucher alle Lernmaterialien vollständig herunterladen.

Als weitere Limitation ist zu nennen, dass Auszubildende aufgrund von begrenzten zeitlichen und finanziellen Ressourcen nicht in das Projekt einbezogen werden konnten. Ein entsprechender Fokus im Rahmen nachfolgender Projekte wäre von Relevanz, um die Unterrichtsmaterialien von den Auszubildenden selbst evaluieren zu lassen und um zu prüfen, ob diese, aus gesundheitswissenschaftlicher Perspektive herausfordernde Zielgruppe (i.d.R.: jung, gesund, überwiegend männlich) mit den Materialien erreicht und eine Änderung von Einstellungen und Verhaltensweisen initiiert werden kann.

Zusammenfassend ergeben sich zahlreiche Ansatzpunkte für weiterführende Forschung. Die Ergebnisse der Fokusgruppendiskussionen sollten mit einer größeren Stichprobe überprüft werden. Ferner erscheint es sinnvoll, die Materialien von Lehrkräften auch nach Erprobung in der Praxis, zu evaluieren und erneut zu modifizieren. Eine Erprobung und Evaluation mit Auszubildenden könnte Aufschluss darüber geben, ob und welche Effekte die Unterrichtsmaterialien aufdie Auszubildenden haben, z. B. ob sich deren Risikowahrnehmung verändert, ein Wissenszuwachs zu verzeichnen ist, sich das Hautschutzverhalten verändert und sich dies in einer unterschiedlichen Inzidenz von Hautveränderungen widerspiegelt.

\section{Fazit für die Praxis}

- Adäquates Hautschutzverhalten sollte bereits beim Erlernen eines Berufes in der Ausbildung initiiert werden.

- Zu Prävention berufsbedingter Hauterkrankungen bei der Verarbeitung von Epoxidharzen wurden Unterrichtsmaterialien für die Berufsschule entwickelt.

- Die Bedürfnisse der Zielgruppe „Lehrkräfte", die diese Materialien nutzen sollen, sind zu berücksichtigen.

- Im Rahmen von Fokusgruppendiskussionen wurden von den Lehrkräften folgende Anforderungen an fremd erstellte Materialien formuliert: methodische Variabilität, Flexibilität und Veränderbarkeit, Exemplarität, Alltagsnähe, Berufsbezug sowie die Berücksichtigung des curricularen Rahmens der Berufsschule.

- Diese Anforderungen wurden bei der didaktischen Konstruktion von Materialien berücksichtigt und die Materialien an die curricularen Rahmenbedingungen (z. B. den handlungsorientierten Unterricht) der Berufsschule angepasst.

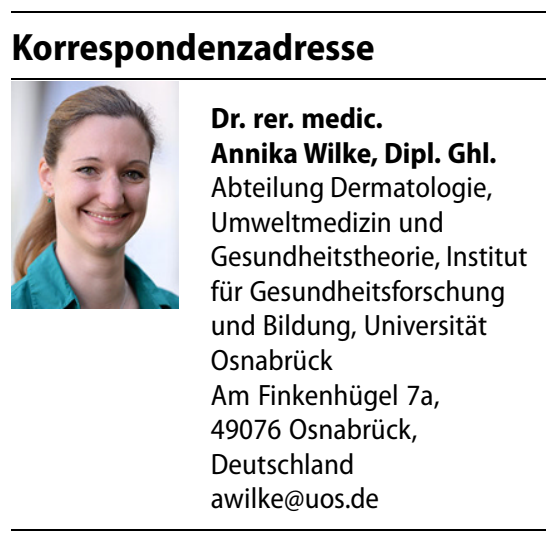

Danksagung. Wir bedanken uns beim Arbeitskreis Epoxidharze für inhaltliche Beiträge und bei Eva Maria Weber für Unterstützung bei der Durchführung der Fokusgruppendiskussionen.

Förderung. Das Projekt „EpoxSafe@School“ wurde von der Deutschen Gesetzlichen Unfallversicherung (DGUV) gefördert (Projekt-Nr. FF-FP 0401).

Funding. Open Access funding provided by Projekt DEAL. 


\section{Einhaltung ethischer Richtlinien}

Interessenkonflikt. A. Wilke, A. Hansen, M. Ludewig, M. Rocholl und S.M. John geben an, dass kein Interessenkonflikt besteht.

Die Fokusgruppendiskussionen wurden gemäß Runderlass des Niedersächsischen Kultusministeriums vom 01.01.2014 „Umfragen und Erhebungen in Schulen" (SVBI. 2014, S. 4) nach Zustimmung der zuständigen Niedersächsischen Landesschulbehörde unter Wahrung der datenschutzrechtlichen Vorschriften durchgeführt. Von allen an den Fokusgruppendiskussionen beteiligten Personen liegt eine schriftliche Einverständniserklärung vor.

Open Access Dieser Artikel wird unter der Creative Commons Namensnennung 4.0 International Lizenz veröffentlicht, welche die Nutzung, Vervielfältigung, Bearbeitung, Verbreitung und Wiedergabe in jeglichem Medium und Format erlaubt, sofern Sie den/die ursprünglichen Autor(en) und die Quelle ordnungsgemäß nennen, einen Link zur Creative Commons Lizenz beifügen und angeben, ob Änderungen vorgenommen wurden.

Die in diesem Artikel enthaltenen Bilder und sonstiges Drittmaterial unterliegen ebenfalls der genannten Creative Commons Lizenz, sofern sich aus der Abbildungslegende nichts anderes ergibt. Sofern das betreffende Material nicht unter der genannten Creative Commons Lizenz steht und die betreffende Handlung nicht nach gesetzlichen Vorschriften erlaubt ist, ist für die oben aufgeführten Weiterverwendungen des Materials die Einwilligung des jeweiligen Rechteinhabers einzuholen.

Weitere Details zur Lizenz entnehmen Sie bitte der Lizenzinformation auf http://creativecommons.org/ licenses/by/4.0/deed.de.

\section{Literatur}

1. Andrees V, John SM, Nienhaus A et al (2020) Economic evaluation of a tertiary prevention program for occupational skin diseases in Germany. Contact Dermatitis. https://doi.org/10. 1111/cod.13506

2. Blümel S, Lehmann F (2015) Zielgruppen, Multiplikatorinnen und Multiplikatoren. In: Bundeszentrale für gesundheitliche Aufklärung (BZgA) (Hrsg) Leitbegriffe der Gesundheitsförderung https:// doi.org/10.17623/BZGA:224-i128-1.0. Zugegriffen: 15. Apr. 2020

3. Craig P, Dieppe $P$, Macintyre $S$ et al (2008) Developing and evaluating complexinterventions: the new Medical Research Council guidance. BMJ 337:a1655

4. Deutsche Gesetzliche Unfallversicherung (2019) DGUV-Statistiken für die Praxis 2018. Aktuelle Zahlen und Zeitreihen der gewerblichen Berufsgenossenschaften und Unfallversicherungsträger der öffentlichen Hand. https://publikationen. dguv.de/widgets/pdf/download/article/3673. Zugegriffen: 15. Apr. 2020

5. Diepgen T, Dickel H, Becker D et al (2002) Beurteilung der Auswirkung von Allergien bei der Minderung der Erwerbsfähigkeit im Rahmen der BK5101. Teil I: Acrylate/Methacrylate, EpoxidharzSysteme, Formaldehyd, Dichromat, Kolophonium,
Latex, Nickel, p-Phenylendiamin. Dermatol Beruf Umwelt 50:139-154

6. Döring N, Bortz J (2016) Forschungsmethoden und Evaluation in den Sozial- und Humanwissenschaften. Springer, Berlin, Heidelberg

7. Dresing T, Pehl T (2018) Praxisbuch Interview, Transkription \& Analyse. Anleitungen und Regelsysteme für qualitativ Forschende. Dr. Dresing und Pehl, Marburg

8. Higgins C, Cahill J, Jolanki R et al (2020) Epoxy resins. In: John SM, Duus Johansen J, RustemeyerT, Elsner P, Maibach HI (Hrsg) Kanerva's occupational dermatology. Springer, Cham, S757-788

9. John SM, Duus Johansen J, Rustemeyer T et al (Hrsg) (2020) Kanerva's occupational dermatology. Springer, Cham

10. Kultusministerkonferenz (2007) Handreichung für die Erarbeitung von Rahmenlehrplänen der Kultusministerkonferenz für den berufsbezogenen Unterricht in der Berufsschule und ihre Abstimmung mit Ausbildungsordnungen des Bundes für anerkannte Ausbildungsberufe. https:// www.kmk.org/fileadmin/veroeffentlichungen beschluesse/2007/2007_09_01-Handreich-RIplBerufsschule.pdf.Zugegriffen: 15.Apr. 2020

11. Kultusministerkonferenz (2015) Rahmenvereinbarung über die Berufsschule. Beschluss der Kultusministerkonferenz vom 12.03.2015. https://www.kmk.org/fileadmin/Dateien/ veroeffentlichungen_beschluesse/2015/2015 03_12-RV-Berufsschule.pdf. Zugegriffen: 15. Apr. 2020

12. Lamnek S, Krell C (2016) Qualitative Sozialforschung. Beltz, Weinheim

13. Lau MY, Matheson MC, Burgess JA et al (2011) Disease severity and quality of life in a followup study of patients with occupational contact dermatitis. Contact Dermatitis 65:138-145

14. Lenz M (2011) Komplexe Interventionen. Notfall Rettungsmed 15:245-246

15. Mayring P (2010) Qualitative Inhaltsanalyse Grundlagen und Techniken. Beltz, Weinheim

16. Meisert A (2012) Wie kann Biologieunterricht geplant werden? In: Spörhase U (Hrsg) Biologiedidaktik. Cornelsen, Berlin, S241-272

17. Niedersächsisches Kultusministerium (2001) Materialien für Lernfelder für die Berufe des Bereichs der Humandienstleistungen sowie für die Berufsfelder Ernährung und Hauswirtschaft, Agrarwirtschaft und Körperpflege. https://nibis. de/uploads/2bbs-poelking-oesselmann/files/ MAT\%20Lernfelder-2001.pdf. Zugegriffen: 15. Apr. 2020

18. Paulus $P$ (2015) Schulische Gesundheitsförderung - vom Kopf auf die Füße gestellt. Von der Gesundheitsfördernden Schule zur guten gesunden Schule. https://www.gesunde-schulen-zuerich.ch/ globalassets/gesundeschulenzuerich/dateien/ manuskript_schulische_gesundheitsfoerderung. pdf. Zugegriffen: 15. Apr. 2020

19. Paulus P, Dadaczynski K (2015) Gesundheitsförderung und Schule. In: Bundeszentrale für gesundheitliche Aufklärung (BZgA) (Hrsg) Leitbegriffe der Gesundheitsförderung https://doi.org/ 10.17623/BZGA:224-i051-1.0

20. Pelz C, Schmitt A, Meis M (2004) Knowledge Mapping als Methode zur Auswertung und Ergebnispräsentation von Fokusgruppen in der Markt- und Evaluationsforschung. Forum Qualitative Sozialforschung 5(2):35

21. Reetz L, Seyd W (2006) Curriculare Strukturen beruflicher Bildung. In:Arnold R, Lipsmeier A(Hrsg) Handbuch der Berufsbildung. VS, Wiesbaden, S227-259
22. Schlicht W, Zinsmeister M (2015) Messen, bewerten, beschreiben, evaluieren. In: Schlicht W, Zinsmeister M (Hrsg) Gesundheitsförderung systematisch planen und effektiv intervenieren. Springer, Berlin, Heidelberg, S89-107

23. Schulz M (2012) Quick and easy!? Fokusgruppen in der angewandten Sozialwissenschaft. In: Schulz M, Mack B, Renn O (Hrsg) Fokusgruppen in der empirischen Sozialwissenschaft. Von der Konzeption bis zur Auswertung. Springer, Wiesbaden, S9-22

24. Schwarzer R (2004) Psychologie des Gesundheitsverhaltens. Einführung in die Gesundheitspsychologie. Hogrefe, Göttingen

25. Spee T, Timmerman JG, Ruhl R et al (2016) Determinants of epoxy allergy in the construction industry: a case-control study. Contact Dermatitis 74:259-266

26. Suuronen K, Back B, Aalto-Korte K et al (2019) Skin exposure to epoxy chemicals in construction coating, assessed by observation, interviews, and measurements. Contact Dermatitis 80:18-25

27. Wilke A, Hansen A, Ludewig M et al (2018) Abschlussbericht zum Vorhaben „Prävention von Berufsdermatosen bei Auszubildenden mit Kontakt zu Epoxidharzen (EpoxSafe@School 1.0)“ (FF-FP 0401). https://www.dguv.de/projektdatenbank/ 0401/ab_29.06.2018_fp401.pdf.Zugegriffen: 15. Apr. 2020 